\title{
System of Design of Universal Logo Should Be Adapted to the Need of Development of Modern City
}

\author{
Xinsheng Guo \\ Zhengzhou University of Light Industry \\ Zhengzhou, China 450002
}

\begin{abstract}
City logo refers to signal, symbol and so on, for instructions, discrimination and guidance in city. With the development of modern cities, as well as the increasing of international exchanges, design of universal Recognition of city should develop towards the direction of international standardization. By maximizing the unified design, enable people to reach a consensus in information of public recognition, to make up the obstacles brought by different cultures, different languages, different geographic disparities, so that people's activities will be more convenient.
\end{abstract}

Keywords-design of city logo; symbol; information; standardization

\section{INTRODUCTION}

With the rapid development of China's economic construction, urban construction is changing with each passing day. Urban roads are expanded unceasingly, and architectural scale is enlarged constantly, and new blocks and new roads are formed from time to time, all these has fully reflected the achievement of economic construction in our country, and demonstrated the achievements of reform and opening up policy and the advantages. Urban development has many objective reasons, such as the sustained economic prosperity, the rapid expansion of urban population, the rapid increase in transport vehicles, and the continuously increasing in population mobility and other factors. While promoting the development of urban construction, these factors also cause the disorder and confusion of urban traffic order, increasing burden to urban management system, thus bring about negative impact on the development of urban construction. It can be seen from the economically advanced countries in the world that, modern urban construction not only embodies the superficial forms of high-rise buildings and crisscrossed road interchanges, but also reflects in whether or not people who live in the city feel convenient to travel. Traffic efficiency is a very important aspect to investigate modern city. It is not uncommon for Chinese people to ask the way, but it is few in foreign countries. Here we do not exclude the factors of habits and individual character, but I think this kind of habit formation is directly related to the perfection of universal logo system construction in the city.

Tokyo is the city with highest percentage of the population in the world. But people who have been to Tokyo feel the traffic is very convenient with perfect order. There is an important factor in addition to its well-developed metro system, namely the perfect universal logo system in this city: various logos are made from aboveground to underground, whether the blocks, intersection, or park, shopping malls, the logos are quite completed, making people amazing, reflecting the comprehensive ability of an advanced city in the world. There is still a wide gap between urban constructions in China with that in Tokyo. I'd like to talk about some superficial views below regarding the development of urban universal logo design system.

\section{CONCEPT OF CITY LOGO}

Sign is also called "symbol", "mark" or the "indicating signal". From the perspective of semiology, it is a symbol of using one object to represent another one, "it is not only the material object as well as the psychological effect". [1] Symbols have two aspects of function, namely "Signifier" and "Signified". Signifier refers to the existence form of the symbol itself, namely the physical function, and Signified refers to the meaning represented by the symbol, namely the psychological instruction function. City logo refers to the mark, symbol, and signal and so on used for instructions, discrimination and guidance in the aspects of direction, roads, and facility utilization in city, "it is the form and practice to express the message to be conveyed in the form of marks." [2] Its main form is to convey information in the visual and symbolic way, but it also includes the content of hearing, sense of touch, etc. broadly. For example, the traffic signs and floor lamps that are commonly seen are visual symbols; there is voice prompt in some cities' sidewalks, and that is auditory sign; there are still the logos in terms of touch designed for the blind in public place. In broad scope, all the symbols that can play the role of instruction and guidance can be called city logo. For example, Pearl Tower is the landmark building of Shanghai and the city symbol of Shanghai; urban traffic lights are the traffic signs; there is general identification of "Emergency Exit" at fire stair between the floors and so on.

\section{TYPES OF UNIVERSAL CITY LOGO}

\section{A. Classification of Universal City Logo}

There are international, national, municipal and regional logos if divided by application scope.

There different types of transportation, road, commerce, leisure, sports and life from the application functions. 
There are such types as flat, three-dimensional, photoelectric, and sound, touch and other integrated types form the manufacturing types.

There are such forms as text, pictures, symbols and device and other forms from the expression forms. mode.

There are natural and artificial types from the existence

Thus it can be seen that city logo system is an important part of urban public facilities construction that is closely related to people's daily life, especially in the densely populated cities and the region with highly developed economy, urban environment has become a complex space with high density and multi-level, while the contact between people is reducing gradually. Especially with the improvement of material life and the upcoming aging society, we shall also pay attention to the design targeting the aging class, and the factors of understanding and recognition ability of children, the disabled and other vulnerable groups and so on.

\section{MAIN ENVIRONMENTAL CONDITIONS FOR UNIVERSAL LOGO TO BE USED IN THE CITY}

\section{A. Urban Symbols and Universal Design}

With the development of urbanization, modern urban construction not only needs to improve the traffic and environmental conditions, symbolic architectural symbols also need to be constructed for urban construction. Especially in some cosmopolitan cities, some landmark buildings are often constructed in the process of urban construction to improve city image, such as the Oriental Pearl of Shanghai, Tokyo Tower in Tokyo, and New York's Times Square, etc. In a sense, the symbolic buildings have already become the fixed identity concept in people's mind, for instance, if these buildings appear in the close-up shots of a movie, then people will immediately realize where the story takes place, and this is the special features of city symbol. In addition, we can also see some universal logos in some public places that are frequently visited internationally, such as international airport, railway stations, subway and so on, such as the bathroom, baggage, exits, emergency channel and fire protection measures etc., these are often used in the internationally unified form of design, so that it is convenient for people from different countries speaking different languages to identify and use, thus to form the standard system of universal logo.

\section{B. Roads and Outdoor Signs}

Urban road traffic and outdoor logo is one of the universal logos that are used most frequently and most closely related to urban construction. For example, the design of Tokyo subway is very clear and humanized, although there are various crossover subway lines, passengers can easily find the information such as position of each station, prices needed for the tickets, interchange locations etc. on the map, so there is no need for passengers to ask the way, which has improved the efficiency of urban traffic. The same is true for urban roads' instructions, which have two guidance systems for vehicles and pedestrians. Vehicle speed is relatively fast in the process of driving, and it has higher requirements for the accuracy of recognition system at intersections. The distance to next intersection is indicated on road signs in some countries, so that the driver can make a choice within easy distance, to avoid causing traffic congestion or traffic accidents. Pedestrians require the contents of indication system to be detailed and precise in addition to be striking. The streets that have been changed in the process of urban construction shall be updated in time, and the surrounding significant environment or building shall be indicated and so on. For example, the street maps of some cities are not designed in accordance with the standard map direction (with north at the top), but designed according to the actual position of the viewer, which is convenient and practical and has greatly improved the viewer's judging ability, indicating that the designers design according to the actual needs, which is quite instructive. Moreover, the location, position, angle, light, color, font, size and other details shall also be considered in the design of indicating system such as road sign. Only many aspects' coordination and integration can be made, can the road indication system be able to play its role truly.

\section{Public Facilities System}

Urban public facilities are the publicities or equipment set up in the main places for the public activities in city, to use the venue, equipment, devices, etc. of these facilities correctly, reasonably and safely, the concise, clear and straightforward identification system is needed for propaganda, popularization and tips. The requirements for prompt system regarding prevention of injuries, damages and dangers and other emergencies are higher, and the three basic principles of easy to identify, easy to understand, and easy to operate shall be satisfied, in order to play the role of guidance in emergency situations timely, to reduce the losses, to provide safety guarantee for city life. The prompt system of other aspects such as environmental protection, energy saving, personnel flow, facilities using conditions (special equipment for disabled) shall also be perfect and complete as far as possible, so that the public facilities system can be used reasonably.

\section{Identification System in Crowded Environment}

Historical site, park, museum, stadium, hospital and university campus are the special places with crowded people and higher frequency of people movement especially in some fixed time. For example, at the time of entering or exiting from the large stadium where performance or competition is hold, or the parks at holiday, or the breaking time of university, the degree of crowdedness will achieve its peak, and the order is quite chaos. So it is very important and critical to split them reasonably and use the public facilities orderly using environment identification design to avoid chaos or accident. With the perfect visual design and guidance system, people can quickly judge and use, which will greatly improve the efficiency. People will gradually be familiar with and grasp the meaning and function of these logos after many times' contacting and using, thus people will rely on and accept logo system, and their behavior consciousness in public environment will be improved gradually, to create a more harmonious and comfortable public environment. 


\section{E. Identification System in Closed Environment}

Enterprises and residence communities belong to the relatively closed environment relative to other urban environment. Relatively independent environment and relatively fixed personnel is the characteristics closed environment. The main characteristics of identification system in such environment are systematic, practical, beautiful and permanent. Some large enterprises have specialized enterprise image recognition system design (VIS), to mainly highlight the fixed identification system such as enterprise logo, special color for the enterprise, and enterprise standard font, playing a good role in unified visual effect and enterprise propaganda, and obtaining good effects in terms of improving enterprise image and strengthening enterprise cohesion, etc..

\section{F. Other Environment}

Shops, markets, and urban villages have unique environment, so the guidance system shall also be designed according to their actual situation.

\section{FACTORS INFLUENCING URBAN UNIVERSAL LOGO DESIGN}

\section{A. Environmental Factors}

Urban construction is accelerated dramatically in recent years, and the rapid urban population expansion has made the original urban design system by far cannot satisfy the use function. As a result, cities began to develop toward air and underground multi-level space. The large scale urban construction and road transformation has made the original city beyond all recognition, those existing landmark buildings also gradually disappear and replaced by the buildings with more complete function, more reasonable structure and more beautiful appearance, followed by the logo system update. Although people always try to use all kinds of measures to deal with various problems, the difficulty of road recognition will increase virtually, and people who live in this city depending on experience have to choose again.

\section{B. Policy Factors}

The influence on urban changes by environmental factors can be said as a progressive process, yet it is the role of policy factors that make the urban environment changes dramatically. For example, when the space environment in some mediumsized cities cannot be expanded greatly completely, the government usually takes large scale transfer policy, such as Shanghai Pudong, Zhengzhou new east area, etc., a administrative region rises rapidly within a short time, to solve the deficiency of urban development fundamentally, which is also one of the characteristics of modern urban construction. In the construction of the new urban district, roads may be wider and population density is relatively rare, which is more likely to lead to accidents, or new problems will rise due to imperfect urban logo or untimely supporting.

\section{Disaster Factors}

Disaster factors mainly include unpredictable disasters (such as fire, unrest and war, etc.) including natural disasters.
Natural disasters are often aggressive, having huge destructive power within a short period, and people tend to lack of protective ability against these disasters. As a result, people shall be educated regarding the measures of evacuation and salvation in the city prior to the disaster's coming, and the cognition of these logos and common sense for use shall be widely publicized, for salvation and self-salvation in emergency situation, to avoid causing a greater loss.

\section{BASIC CHARACTERISTICS OF UNIVERSAL CITY LOGO DESIGN}

\section{A. Unity of Design}

City logo system is a kind of visual symbol used in the overall situation having the role of guidance, and the overall design should be unified. The overly complex graphics, the excessive design style, too many colors or the modeling of too many changes is bound to affect the basic guidance and instruction function of the city logo system, resulting in the negative results such as visual identity confusion and being difficult to remember. Therefore, unity of design is the first principle of city logo design, which can only play the effects of aesthetic and decoration by paying attention to the general principle of unity.

\section{B. Specific Instructions}

City logo is the identification symbol of experiential identity and cognition facilitating people's travel. Therefore, it shall be suitable for the recognition and cognition of all people of different ages, different culture, and from different regions. Pay attention to take easy identification as the basic principle, as the too obscure graphics, the too small words and the too complicated colors will greatly reduce its function of use. Therefore, its feature of instruction in city identity system shall be grasped first of all. For instance, the decreased vision of the elderly, children's understanding ability of graphics, differences in cultural level and foreigners' understanding ability of Chinese language are all the factors to be considered.

\section{Rationality of the Environment}

The street and rode condition in city is complex, especially for the foreigners who are unfamiliar with environment, the rationality of logo setting environment is particularly important, otherwise, its convenience and functionality will lose. Height, size, color, light and location are all related to the environment. Therefore, it shall set according to circumstances of the environment, and select the most reasonable location and the most reasonable way.

\section{Completeness of the Content}

Urban structure is diverse, and one of the characteristics of modern city is to develop to the multi-space and compounding form. And the three-dimensional and rapid urban traffic is bound to bring traffic complexity and differentiation. To make city identification more adapt to these characteristics, content completeness shall be strengthened correspondingly. Now, we can see the viewer's current location in the map in city's street, which is a great improvement. In England, you can find the residents' house number clearly marked on the signboard in 
front of their door, thus you can find the residents' location easily. As a result, only the complete recognition system can be able to meet the requirements.

\section{BASIC PRINCIPLES OF UNIVERSAL CITY LOGO DESIGN}

\section{A. Artistry and Identification}

On the one hand, as the striking guidance carrier, city logo plays the important visual role in urban environment, which is a kind of symbolic identification symbol, but also part of the landscape design in the city. Therefore, logo design shall not only consider its recognition, but the artistic and aesthetic factors shall also be considered, and be coordinated with the urban environment and people's aesthetic habits. Thus it is consistent with green design concept that we advocate. Designers shall try their best to make city logo be the scenery in the city, rather than the ugly visual garbage.

On the other hand, the ultimate goal of logo design is to convey the identification information, and facilitate people's travel and life, which cannot simply pursue the aesthetic effect but ignore the function. The design should be easy to identify and understand. "The basic function of symbol is cognition and communication" [3], said by Hideya Hebei, a Japanese designer who has successfully designed the guidance system for Tokyo metro, "I try to make these subway lines be understood even by the grandpa and grandma from rural areas", [4] I think this is probably the best statement on the standard of city logo design. Mutual combination of artistry and practice is the principle always advocated by modern design.

\section{B. Adaptation and Scientificity}

"Helping people" and "convenience" are the design motives mentioned by Hideya Hebei. We can see that the ultimate goal of urban recognition design is to meet the needs of peoples' application requirements, to provide services to urban life and operating. Therefore, a wider range of objects and the scope shall be considered in the process of design, especially the elderly, children, the disabled and other social vulnerable groups, the scale, site, color, material science and rationality shall be considered. As for the interactional relationship between environment and psychology, city logo design shall be considered with scientific attitude as a whole. Only in this way, can the urban environment logo system play its biggest role.

\section{Green Design and Environmental Protection Concept}

Design is to serve the people, and city logo design is a design for people. Here, we shall pay attention to the overall concept of green design: on the one hand, the renewable and recyclable applied materials with low energy consumption and pollution-free shall be valued, to avoid waste, excessive consumption and damage to the environment, to achieve the goal of green environmental protection as far as possible; on the other hand, we shall pay attention to the influence on people's vision and psychological feeling by design. The design just pays attention to the use function without valuing human feelings cannot be accepted and used by people. Only the design that is able to satisfy people's aesthetic habits and the aesthetic psychology of the masses can truly play its role. As a result, green design is the concept of a broader level, which is one of the basic principles to be mastered by modern designers.

\section{Prospect of UniVERSAl City Logo Design}

\section{A. Smart Technology}

With the coming of information era, more new technology, new material and new concept will be used in city logo design. Especially with the development of electronic technology, the new technology supported by network technology will make greater changes in the urban identification system. Intelligent, accessibility, easy operation and maintenance will be the direction of future development. Especially, the improvement and research will be made further for identity disorders caused by time factor and light factor. The progress of science and technology will inevitably bring the progress of applied technology. The city logo system in future will become more flexible and convenient.

\section{B. Internationalization of Information}

The Public Logo Standards published by Japan said: "the graphic guidance used in public places with unspecific majority of people shall be understood when people see them, and can be used skillfully according to the theme of the subjects, which are very easy to understand," [5] this is the best understanding of design. The starting point of this research is to realize domestic or international general standard.

Internationalism and standardization are the concept advocated by current universal design, and it is a more open and more macro thinking under the concept of "big world". By the unified design to the largest extent, people may achieve consensus in terms of public recognition information, to make up the obstacles brought by different culture, different language and different regions, making people's activities more convenient.

\section{Humanization of Design}

Humanized design is an important characteristic of the information age. The design concepts of "fair use", "flexible use", "body burden as little as possible" will be expanded further, and city logo design will develop toward the aspects that can meet wider population's use requirement, interest and ability. Relationship between people and logo will be more harmonious, and logo design environment will be more humanized and rationalized in terms of graphic design, material application, logo size, setting environment, etc. People's personality and nature will get more respect. More attention will be paid to interactive relationship between people and logo, and the urban environment will also develop to be more beautiful, harmonious and sustainable.

\section{CONCLUSION}

With the increase of international exchanges, the world is more and more like a big home and people hope to enhance mutual understanding through more mutual exchanges. With the increase of these communication activities, our city will 
establish a more extensive and more closely connection with other cities. Under the influence of world economy, culture, science and technology globalization, the reform in China will be deepened, and the speed of urbanization will be further accelerated. With the development of urban construction, we need to constantly improve our understanding and take a new look at the setting of current city logo system, to provide convenient, quick and accurate service for more people. This is not only to adapt to the international trend, at the same time for ourselves.

\section{REFERENCES}

[1] Written by Naoto Tanaka and others, translated by Wang Baogang, Guo Xiaoming. Universal Design of Logo Environment [M].Beijing: China Architecture \& Building Press, 2004.3.

[2] Keiko Hirano. Era of Icon 1950-2004 [M]. Tokyo: Rikuyosha, 2004.9.

[3] Written by Editorial Board of Standard Information Symbols Popular Edition. Guide Book of Standard Information Symbols [M]. Tokyo: Taisei Publishing House, 2001.12.

[4] Chen Zongming, Huang Huaxin. Introduction to Semiotics [M]. Zhengzhou: Henan People's Publishing House, 2004.12.

[5] Ling Jixiao. Fifteen Lectures of Art Design [M], Peking University Press, Beijing, 2006. 